\title{
CLINICAL AND RADIOGRAPHIC EVALUATION OF OSSEODENSIFICATION VERSUS OSTEOTOME FOR SINUS FLOOR ELEVATION IN PARTIALLY ATROPHIC MAXILLA: A PROSPECTIVE LONG TERM STUDY
}

\author{
Shereen W Arafat* and Mohamed A Elbaz**
}

\begin{abstract}
Objectives: The current study was conducted to evaluate crestal sinus floor elevation with either osteotome or osseodensification in posterior atrophic maxilla.

Material \& methods: 24 crestal sinus floor elevations were performed for 24 patients with at least $5 \mathrm{~mm}$ residual bone height. 12 randomly selected patients received osteotome sinus elevation (group 1), and 12 received osseodensification sinus elevation (group 2). The treatment outcome was evaluated at 6,12 months of healing clinically and radiographicaly. Implant 1ry and 2ry stability, marginal bone loss, and bone gain were recorded and statistically analyzed.
\end{abstract}

Results: group 2 showed significantly higher ISQ values immediately postoperatively and at 6 months. There was significant increase of bone height (bone gain) in both groups ( $\mathrm{P}=0.001)$, and bone gain was $2.79 \pm 0.30 \mathrm{~mm}$ and $3.33 \pm 0.25 \mathrm{~mm}$ in group $1 \& 2$ respectively.

Conclusion: Osseodensification sinus floor elevation was superior to osteotome elevation regarding the 1ry \& 2ry stability, and bone gain.

KEY WORDS: osseodensification , osteotome, sinus lift, atrophic maxilla

\section{INTRODUCTION}

The rehabilitation of the edentulous posterior maxilla using osseointegrated implants is often challenging due alveolar bone resorption, low bone density and maxillary sinus pneumatization. Thus, an augmentation procedure is often indicated in this area. Maxillary sinus lift is one of the most common surgical techniques used for increasing the available bone volume to place implants and restore function and esthetics. Lateral approach for sinus lift is usually indicated when residual bone height is less than 5 to $6 \mathrm{~mm} .{ }^{(1,2)}$ While transcrestal approach can be successfully adopted when residual bone height

* Associate Professor of Oral \& Maxillofacial Surgery, MSA University.

** Lecturer of Oral \& Maxillofacial Surgery, MSA University. 
is at least $5 \mathrm{~mm} .^{(3,4)}$ Osteotome sinus floor elevation was $1^{\text {st }}$ introduced by Summers ${ }^{(5,6)} 1994$, and proved to be less invasive, more conservative, less time consuming, and reduces postoperative discomfort to the patient. ${ }^{(7,8)}$ Moreover, this technique was found to yield predictable results, with success rates of at least $95 \%{ }^{(9,10)}$

Osseodensification is a new surgical technique of biomechanical bone preparation performed for dental implant placement where bone is compacted and autografted into open marrow spaces and osteotomy site walls in outwardly expanding directions. ${ }^{(11)}$ It was reported that Osseodensification increases the bone-implant contact, bone density, and primary stability. ${ }^{(12)}$ Moreover, The insertion torque peak is directly related to implant primary stability and host bone density. ${ }^{(13)}$ Furthermore, Ottoni et al. ${ }^{(14)}$ showed a reduction in failure rate of $20 \%$ in single-tooth implant restoration for every $9.8 \mathrm{~N} \mathrm{~cm}$ of torque increased.

The objective of this study was to evaluate crestal sinus elevation using ossedensification versus osteotomy clinically and radiographically in terms of marginal bone loss, primary and secondary stability and bone gain around the implant.

\section{MATERIALS AND METHODS}

This prospective clinical study was conducted at Oral Surgery and Anesthesia Department, Faculty of Dentistry, MSA University. Patients of at least 18 years old requiring 1-2 Implants in the atrophic posterior maxilla with at least $5 \mathrm{~mm}$ residual bone height were enrolled. Patients were excluded from the study if they presented one of the following exclusion criteria: inability to maintain proper level of oral hygiene throughout the study, any medical condition, or medication that might compromise bone healing, and inability to return for followup visits. Every subject has signed an informed consent before entering the study.
The current study sample comprised 24 patients. 17 patients of the 24 were male $(70.8 \%)$, while 7 patients were female (29.2\%) with age range 23-65 years.

\section{Surgical protocol}

Prior to surgery, radiographic evaluation through panoramic radiographs and cone-beam CT scan was performed to identify the residual bone height, width, the bone quality at the surgical site, and to determine the sequence of bone drills for implant site preparation. Local anesthesia articaine chloridrate 4\% with adrenaline 1:100 000 (Alfacaina N, Weimer Pharma, Rastat, Germany) was administered. A fullthickness mucoperiosteal flap was elevated.

Patients were randomly divided into 2 groups. Group 1 received osteotome sinus lift and simultaneous implant placement, and group 2 received osseodensification sinus lift and simultaneous implant placement. In osteotome sinus lift cases, bone drilling was performed with conventional drills with working length 1 $\mathrm{mm}$ shorter than the residual bone height. Then osteotome (Straumann AG) was used with Light careful tapping using a mallet to elevate the Schneiderian membrane. The osteotomy was subsequently enlarged. A Valsalva maneuver was performed to verify the sinus membrane integrity, and tapered Screw Plant* implants were inserted.For osseodensification group, drilling was performed within an approximate safety zone of $1.0 \mathrm{~mm}$ from the sinus floor using a pilot drill, then the narrowest Densah ${ }^{\circledR}$ Bur (2.0). The motor was then changed to reverse - Densifying Mode (Counterclockwise drill speed 800-1500 rpm with copious irrigation), and osteotomy was created. Pressure with a pumping motion was performed to reach the sinus floor. The next wider Densah ${ }^{\circledR}$ Bur (3.0) was then used and advanced into the previously created osteotomy with modulating pressure and a pumping motion.

\footnotetext{
* Spectra-System ${ }^{\circledR}$, ScrewPlant, Implant DirectTM LLG, Malibu hills, USA
} 
When feeling the haptic feedback of the bur reaching the dense sinus floor, modulating pressure with a pumping motion was continued to advance past the sinus floor in $1 \mathrm{~mm}$ increments. Maximum possible advancement past the sinus floor at any stage did not exceed $3 \mathrm{~mm}$. Bone was pushed toward the apical end and began to gently lift the membrane and autograft compacted bone. Tapered Screw Plant implants then were inserted. (Fig 1)

At the time of implant placement, resonance frequency analysis was performed to record the implant stability quotient (ISQ) value. The transducer (Smartpeg; Integration Diagnostics AB, $\mathrm{G} €$ oteborg, Sweden) was connected to the implant, and the analyzer probe was located close to the Smartpeg, and ISQ value was given by the Osstell device. The flap then was repositioned and sutured with 3-0 non-resorbable sutures.

The surgical area was maintained prosthesis free. Postsurgical instructions were provided to avoid infection, control bleeding and to avoid suture detachment during the first healing period. Final prosthesis was delivered after 6 month healing period.

Follow-up visits were scheduled after 10 days for suture removal, 1 month for clinical observation,
6 months after surgery for the definitive prosthesis and stability $2^{\text {nd }}$ record, and 6 months after loading for radiographic evaluation.

\section{Clinical and Radiological Parameters}

The implant survival rate was recorded according to Buser et al ${ }^{(15)}$ and Cochran et al ${ }^{(16)}$ which are no pain or any subjective sensation, no clinically detectable implant mobility, no continuous radiolucency around the implant, and no recurrent peri-implant infection.

Cone beam $\mathrm{CT}$ was performed at 6 months postoperatively and at 6 month after loading. The residual bone height before surgery was measured for each planned implant site and was compared with the height attained at 6 months postoperatively. Bone height gain was calculated as the difference between the bone height at 6 months and the residual bone height. Marginal bone height at the mesial and distal aspects were measured and averaged at 6 months postoperatively and 6 months after loading as the distance from alveolar bone crest to the implant end. (Fig 2) The difference between the 2 measurements represented the marginal bone loss. Implant Stability Quotient (ISQ) was measured at the time of implant placement and at 6 months postoperatively.

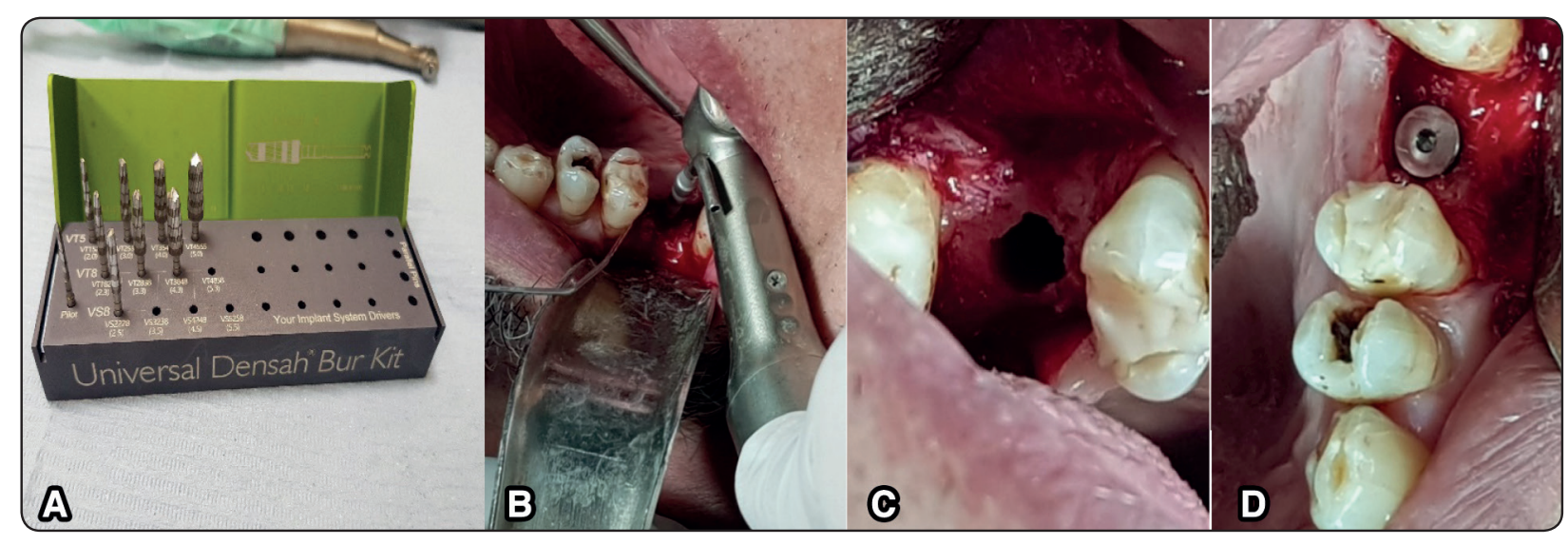

Fig. (1) A) Universal Densah Bur Kit used for osseodensification sinus elevation in group 2, B) drilling the osseodensification site with copious irrigation at the maxillary $1^{\text {st }}$ molar site, C) the prepared osseodensification site after sinus elevation, D) the implant in place at the prepared site after sinus elevation. 
Evaluation was obtained by Scanora 3D, with OnDemand 3D App 1.0.10.4304 viewer. Measurements were calculated by an oral radiologist who was blinded to the surgical procedure and the evaluation were made twice with 10 days period interval.

\section{Statistical analysis}

Independent t-test used to compare between tested groups. Dependent t-test used to compare between follow-up period for each group $(\alpha=0.05)$. Data analysis done using SPSS (IBM Corp. Released 2015. IBM SPSS Statistics for Windows, Version 23.0. Armonk, NY: IBM Corp.)

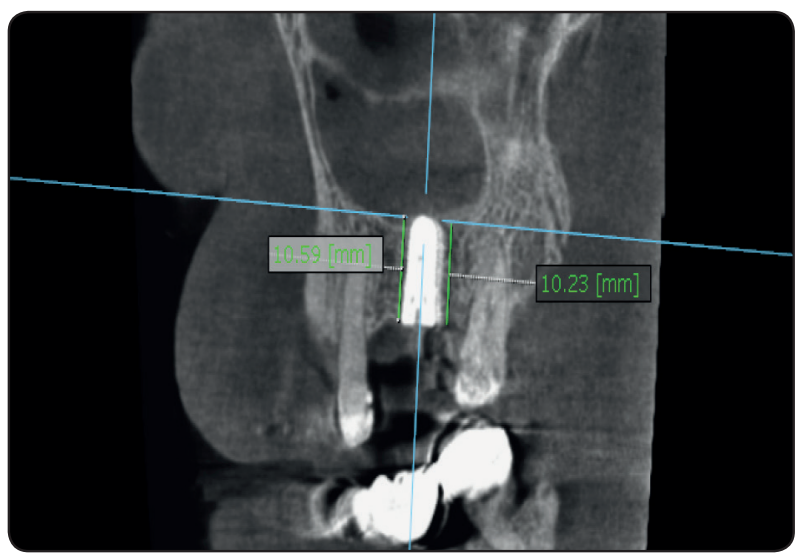

Fig. (2) CBAT image showing the measurement of alveolar bone height mesial and distal to the implant 6 months postoperatively with reference to the implant end for a case of osseodensifcation group.

\section{RESULTS}

All implants osteointegrated successfully in both groups and were clinically stable during abutment tightening at six months postoperatively. Thus, Implant survival rate was $100 \%$ at 6 months from implant loading. No patient complained of pain, and there were no signs of inflammation or Schneiderian membrane perforation.

The pre-surgical bone height in group 1 (osteotome group) was $7.14 \pm 0.47 \mathrm{~mm}$ and in group 2 (osseodensification group) was $7.38 \pm 0.52 \mathrm{~mm}$. The Student's t-test found statistically non-significant difference of pre-surgical bone height between groups $(P=0.260)$. At 6 months postoperatively, there was significant increase of bone height (bone gain) in both groups ( $\mathrm{P}=0.001)$. Comparing the bone gain at 6 months postoperatively between the study groups showed that bone gain in group 2 was significantly higher than bone gain in group 1 . $(\mathrm{P} \leq 0.001)$ which was $3.33 \pm 0.25 \mathrm{~mm}$ and $2.79 \pm 0.30$ $\mathrm{mm}$ respectively. (Fig 3) There was no statistically significant difference between the 2 groups regarding the marginal bone loss at 6 months after loading which was $0.93 \pm 0.15 \mathrm{~mm}$ in group 1 and $0.98 \pm 0.15$ $\mathrm{mm}$ in group 2. $(\mathrm{P}=0.501)$ as shown in table (1)

TABLE (1) Residual bone height, bone gain, marginal bone loss, and implant stability quotient means and standard deviations for the study groups.

\begin{tabular}{|c|c|c|c|c|c|c|}
\hline & & \multicolumn{2}{|c|}{ Osteotome } & \multicolumn{2}{|c|}{ Osseodensification } & \multirow[t]{2}{*}{ p-value } \\
\hline & & Mean & SD & Mean & SD & \\
\hline \multirow[t]{4}{*}{ Residual bone height (mm) } & Immediate & 7.14 & 0.47 & 7.38 & 0.52 & $0.260 \mathrm{NS}$ \\
\hline & 6 Months & 9.93 & 0.57 & 10.71 & 0.43 & $0.001 *$ \\
\hline & p-value & \multicolumn{2}{|c|}{$\leq 0.001 *$} & \multicolumn{2}{|c|}{$\leq 0.001 *$} & \\
\hline & Difference & 2.79 & 0.30 & 3.33 & 0.25 & $\leq 0.001 *$ \\
\hline \multirow[t]{4}{*}{ Stability } & Immediate & 52.83 & 6.29 & 65.17 & 4.39 & $\leq 0.001 *$ \\
\hline & 6 Months & 67.83 & 4.78 & 75.92 & 2.94 & $\leq 0.001 *$ \\
\hline & p-value & \multicolumn{2}{|c|}{$\leq 0.001 *$} & \multicolumn{2}{|c|}{$\leq 0.001 *$} & \\
\hline & Difference & 15.00 & 3.28 & 10.75 & 4.25 & $\leq 0.001 *$ \\
\hline \multicolumn{2}{|c|}{ Marginal bone loss } & 0.93 & 0.15 & 0.98 & 0.15 & $0.501 \mathrm{NS}$ \\
\hline
\end{tabular}




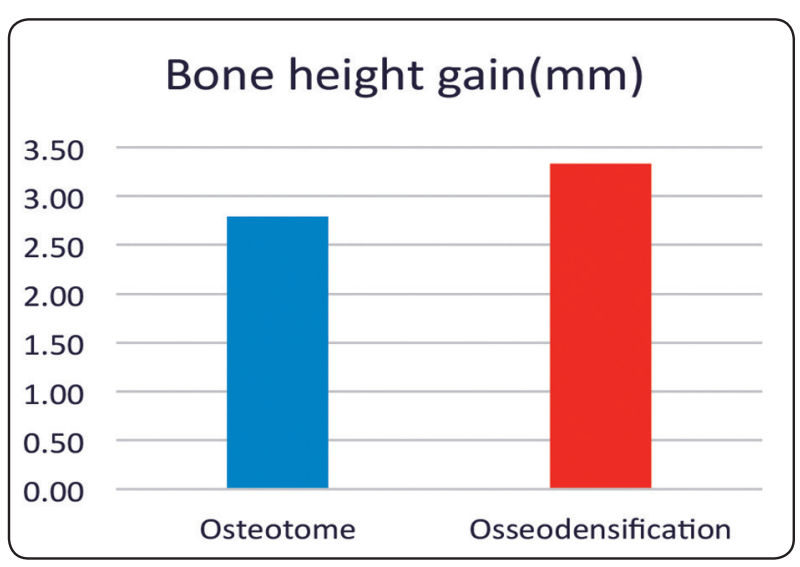

Fig. (3) bone gain in the study groups at 6 months postoperatively.

The mean ISQ value in both groups is shown in figure 4. Both groups showed significant increase in ISQ value from base line (immediately after implant placement) and at 6 months postoperatively $(\mathrm{P} \leq 0.001)$. Comparing ISQ values in both groups was significant at the baseline and at 6 months postoperatively as group 2 showed significantly higher ISQ values at the 2 time intervals.

\section{DISCUSSION}

The main objective of this study was to evaluate crestal sinus floor elevation with either osteotome or osseodensification. A comparison was made of these two techniques regarding the bone gain, marginal bone loss and implant stability. Considering the results of this study, both sinus floor elevation procedures showed $100 \%$ implant survival rate for 6 months after loading. Literature reported that, following use of osteotome technique without bone grafting material, the prognosis may become $97.2 \%$ when the residual bone height is at least $5 \mathrm{~mm} .{ }^{(17)}$

In crestal sinus floor elevation, the achievement of satisfactory implant primary stability is a key factor for osseointegration. In the current study, osseodensification group showed significantly higher ISQ values at the 2 study intervals representing 1ry and 2ry stability. This could be explained by

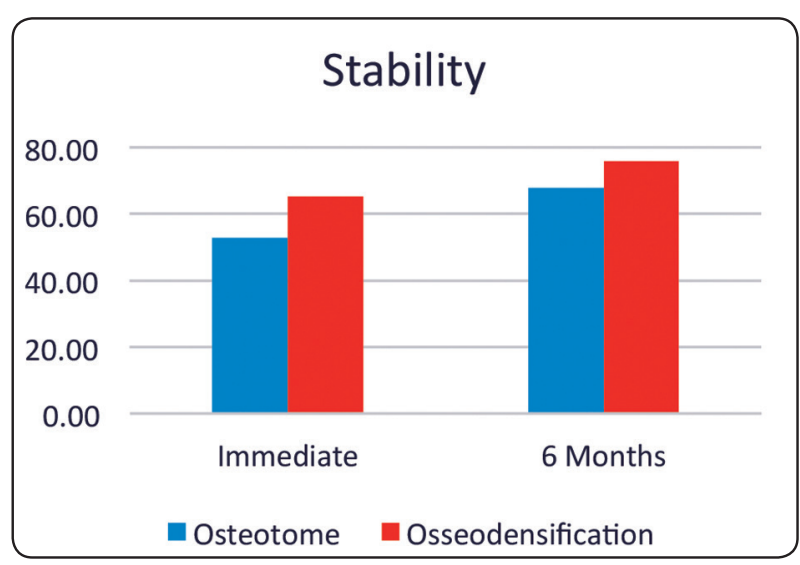

Fig. (4) Implant Stability Quotient ISQ for the study groups immediately after implant placement and at 6 months postoperatively.

the drilling technique of osseodensification, which drives bone compaction in the osteotomy site wall, and the presence of residual bone chips which form an autograft wall around the osteotomy perimeter. This result is consistent with the findings of Jimbo et al ${ }^{(18)}$ in a sheep model. ${ }^{(19)}$ This could be as well an explanation for the finding of significantly higher bone gain in group 2 than bone gain in group 1 at 6 months postoperatively. Moreover, It was concluded in the studies of Frost ${ }^{(20)}$ and Mori et al ${ }^{(21)}$ that the traumatic damage in bone caused by osteotome sinus floor elevation delays the achievement of secondary stability, as increased time needed for the repair of the micro-damage, which stimulates osteoclast activation.

The bone gain in the current study was $2.79 \pm 0.30$ $\mathrm{mm}$ in group 1 , and $3.33 \pm 0.25 \mathrm{~mm}$ in group 2 which is consistent with data reported in Antonaya-Mira and colleagues ${ }^{(22)}$ review of literature.

In the current study, no bone graft was used in the 2 study groups with survival rate of $100 \%$. This finding is consistent with Nedir et al ${ }^{(23)}$ who reported osteotome sinus floor elevation without grafting for sinus augmentation of $3 \mathrm{~mm}$. This was attributed to the osteogenic potential of the shenederian membrane that may give origin for mesenchymal cells that start the osteogenic lineage. ${ }^{(1,24)}$ Moreover, 
it was reported that The implant apex could act as tent-pole that supports the elevated sinus membrane and protect the blood clot. ${ }^{(25-27)}$

Thus, from the results of the current study, it could be concluded that osseodensification sinus floor elevation was superior to osteotome elevation regarding the 1ry \& 2ry stability, and bone gain. Moreover, the use of bone graft could not be essential in the crestal sinus floor elevation using either osteotome or osseodensification procedures.

\section{REFERENCES}

1- Del Fabbro M, Rosano G, Taschieri S. Implant survival rates after maxillary sinus augmentation. Eur J Oral Sci 2008; 116:497-506.

2- Peleg M, Garg AK, Mazor Z. Predictability of simultaneous implant placement in the severely atrophic posterior maxilla: a 9-year longitudinal experience study of 2132 implants placed into 731 human sinus grafts. Int J Oral Maxillofac Implants 2006; 21:94-102.

3- Emmerich D, Att W, Stappert C. Sinus floor elevation using osteotomes: a systematic review and meta-analysis. J Periodontol 2005; 76:1237-1251.

4- Del Fabbro M, Corbella S, Weinstein T, Ceresoli V, Taschieri S. Implant survival rates after osteotome-mediated maxillary sinus augmentation: a systematic review. Clin Implant Dent Relat Res 2012; 14 Suppl 1:e159-168.

5- Summers, R.B. A new concept in maxillary implant surgery: the osteotome technique. Compendium of Continuous Education in Dentistry 1994a; 15: 152-160.

6- Summers, R.B. The osteotome technique. Part 3. Less invasive methods in elevation of the sinus floor. Compendium of Continuous Education in Dentistry 1994b; 15: 698-708.

7- Toffler M. The clinical and practical benefits of combining short implants with minimally invasive osteotome sinus floor elevation in the treatment of the atrophic posterior maxilla. Pract Proced Aesthet Dent 2006; 18:185-192.

8- Esposito M, Grusovin MG, Rees J, et al. Interventions for replacing missing teeth: augmentation procedures of the maxillary sinus. Cochrane Database Syst Rev 2010; (17):CD008397.
9- Emmerich D, Att W, Stappert C. Sinus floor elevation using osteotomes: a systematic review and meta-analysis. J Periodontol 2005; 76:1237-1251.

10- Shalabi MM, Manders P, Mulder J, Jansen JA, Creugers NH. A meta-analysis of clinical studies to estimate the 4.5-year survival rate of implants placed with the osteotome technique. Int J Oral Maxillofac Implants 2007; 22:110-116.

11- Huwais S, and Meyer EG. A novel osseous densification approach in implant osteotomy preparation to increase biomechanical primary stability, bone mineral density, and bone-to-implant contact. Int $\mathrm{J}$ Oral Maxillofac Implants 2017; 32: 27-36.

12- B. Lahens, R. Neiva, N. Tovar, A.M. Alifarag, R. Jimbo, E.A. Bonfante, M.M. Bowers, M. Cuppini, H. Freitas, L. Witek, P.G. Coelho. Biomechanical and histologic basis of osseodensification drilling for endosteal implant placement in low density bone. An experimental study in sheep, J. Mech. Behav. Biomed. Mater 2016; 63: 56-65.

13- Trisi P, Berardini M, Falco A, Podaliri Vulpiani M. New osseodensification implant site preparation method to increase bone density in low-density bone: In vivo evaluation in sheep. Implant Dent 2016; 25: 24-31.

14- Ottoni JM, Oliveira ZF, Mansini R, Cabral AM. Correlation between placement torque and survival of single-tooth implants. Int J Oral Maxillofac Implants 2005; 20: 769-76.

15- Buser D, Mericske-Stern R, Bernard JP, et al. Long-term evaluation of non-submerged ITI implants. Part 1: 8-year life table analysis of a prospective multi-center study with 2359 implants. Clin Oral Implants Res 1997; 8:161-172.

16- Cochran D, Buser D, ten Bruggenkate C, Weingart D, Taylor T, Bernard JP, Peters F, Simpson J. The use of reduced healing times on ITI implants with a sandblasted and acidetched (SLA) surface. Clinical Oral Implants Research 2002; 13: 144-153.

17- Del Fabbro M, Corbella S, Weinstein T, Ceresoli V, Taschieri S. Implant survival rates after osteotome-mediated maxillary sinus augmentation: a systematic review. Clin Implant Dent Relat Res 2012; 14(Suppl 1):e159-e168.

18- Jimbo R, Tovar N, Marin C, Teixeira HS, Anchieta RB, Silveira LM, Janal MN, Shibli JA, Coelho PG. The impact of a modified cutting flute implant design on osseointegration. Int. J Oral Maxillofac Surg 2014b;43: 883-888.

19- Lahens B, Neiva R, Tovar N, Alifarag AM, Jimbo R, Bonfante EA, Bowers MM, Cuppini M, Freitas H, Witek L, Coelho PG. Biomechanical and histologic basis of osseodensification drilling for endosteal implant placement 
in low density bone. An experimental study in sheep. J Mech Behav Biomed Mater 2016; 63: 56-65.

20- Frost HM. A brief review for orthopedic surgeons: fatigue damage (microdamage) in bone (its determinants and clinical implications). J Orthop Sci 1998; 3: 272-281.

21- Mori S, Harruff R, Burr DB. Microcracks in articular calcified cartilage of human femoral heads. Arch Pathol Lab Med 1993; 117: 196-198.

22- Antonaya-Mira R, Barona-Dorado C, Martínez- Rodríguez N, Cáceres-Madroño E, Martínez-González JM. Metaanalysis of the increase in height in maxillary sinus elevations with osteotome. Med Oral Patol Oral Cir Bucal 2012; 17:e146-e152.

23- Nedir R, Nurdin N, Vazquez L, Abi Najm S, Bischof M. Osteotome Sinus Floor Elevation without Grafting: A 10Year Prospective Study. Clin Implant Dent Relat Res 2016; 18: 609-617.
24- Srouji S, Kizhner T, Ben David D, Riminucci M, Bianco $\mathrm{P}$, Livne E. The Schneiderian membrane contains osteoprogenitor cells: in vivo and in vitro study. Calcif Tissue Int 2009; 84:138-145.

25- Bruder SP, Fink DJ, Caplan AI. Mesenchymal stem cells in bone development, bone repair, and skeletal regenerationtherapy. J Cell Biochem 1994; 56:283-294.

26- Gruber R, Kandler B, Fuerst G, Fischer MB, Watzek G. Porcine sinus mucosa holds cells that respond to bone morphogenetic protein (BMP)-6 and BMP-7 with increased osteogenic differentiation in vitro. Clin Oral Implants Res 2004; 15:575-580.

27- Lundgren S, Andersson S, Gualini F, Sennerby L. Bone reformation with sinus membrane elevation: a new surgical technique for maxillary sinus floor augmentation. Clin Implant Dent Relat Res 2004; 6:165-173. 